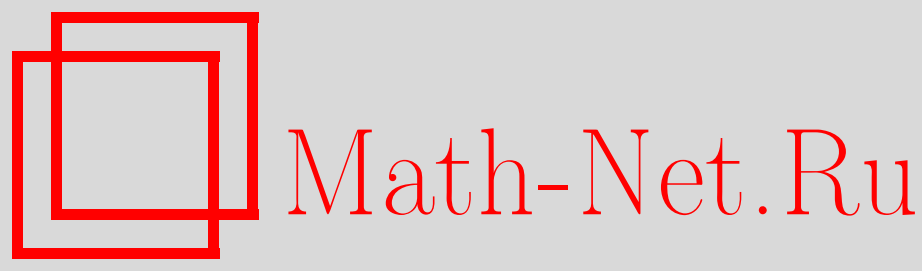

С. Н. Лакаев, А. М. Халхужаев, О числе собственных значений двухчастичного дискретного оператора Шредингера, ТМФ, 2009, том 158, номер 2, 263-276

DOI: https://doi.org/10.4213/tmf6313

Использование Общероссийского математического портала Math-Net.Ru подразумевает, что вы прочитали и согласны с пользовательским соглашением http://www . mathnet.ru/rus/agreement

Параметры загрузки:

IP : 18.209 .158 .208

26 апреля 2023 г., 13:01:52

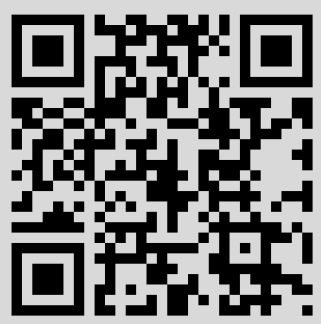




\title{
О ЧИСЛЕ СОБСТВЕННЫХ ЗНАЧЕНИЙ ДВУХЧАСТИЧНОГО ДИСКРЕТНОГО ОПЕРАТОРА ШРЕДИНГЕРА
}

\begin{abstract}
Рассматривается двухчастичный дискретный оператор Шредингера, ассоциированный с гамильтонианом системы двух частиц (фермионов), взаимодействующих только на ближайших соседних узлах. Установлены число и местоположение собственных значений этого оператора в зависимости от энергии взаимодействия частиц, квазиимпульса системы и размерности решетки $\mathbb{Z}^{\nu}, \nu \geqslant 1$.
\end{abstract}

Ключевые слова: гамильтониан, двухчастичный дискретный оператор Шредингера, квазиимпульс, существенный спектр, виртуальный уровень, собственные значения, определитель Фредгольма.

\section{1. ВВЕДЕНИЕ}

В последние годы заметно возрос интерес к исследованию операторов Шредингера (гамильтонианов), описывающих поведение решетчатых квантовых частиц [1].

Кинематика квантовых квазичастиц на решетке довольно своеобразна даже в двухчастичном случае. Например, вследствие того что дискретный аналог лапласиана или его обобщения не являются трансляционно-инвариантными, гамильтониан системы не разделяется на две части: относящуюся к движению центра и связанную с внутренними степенями свободы. Это так называемое явление "избытка масс" для решетчатых систем: эффективная масса $N$-частичного связанного состояния больше, чем сумма эффективных масс квазичастиц, составляющих систему (см., например, [2], [3]).

Двухчастичная проблема на решетке, в отличие от непрерывного случая, когда отделяется движение центра масс, сводится к изучению одночастичной проблемы с помощью преобразования Гельфанда. А именно, гильбертово пространство $\ell_{2}\left(\left(\mathbb{Z}^{3}\right)^{2}\right)$ разлагается в прямой (непрерывный) интеграл фон Неймана, ассоциированный с представлением абелевой (дискретной) группы $\mathbb{Z}^{3}$, образованной с помощью перестановочных операторов на решетке. Тогда двухчастичный гамильтониан также разлагается в прямой (непрерывный) интеграл фон Неймана.

* Самаркандский государственный университет, Самарканд, Узбекистан. E-mail: slakaev@mail.ru

${ }^{\dagger}$ Комплексный научно-исследовательский институт региональных проблем Самаркандского отделения АНРУз, Самарканд, Узбекистан. E-mail: ahmad_x@mail.ru 
В отличие от непрерывного случая соответствующие слойные операторы $h(k)$, $k \in \mathbb{T}^{\nu}$, ассоциированные с разложением прямого интеграла, параметрически зависят от квазиимпульса $k$, который пробегает первую зону Бриллюэна $\mathbb{R}^{\nu} /(2 \pi \mathbb{Z})^{\nu}$. В результате из-за потери сферической симметричности проблемы спектры семейства $h(k)$ оказываются довольно чувствительными к изменению квазиимпульса $k$.

В работе [4] для широкого класса двухчастичных операторов Шредингера $h(k)$, $k \in \mathbb{T}^{\nu}$, ассоциированных с гамильтонианом системы двух произвольных частиц, установлен следующий эффект. Если $\nu$-мерный $(\nu \geqslant 3)$ дискретный оператор Шредингера $h(\mathbf{0}), \mathbf{0}=(0,0, \ldots, 0) \in \mathbb{T}^{\nu}$, имеет виртуальный уровень или собственное значение на нижнем пороге существенного спектра, то при всех ненулевых значениях квазиимпульса $k \in \mathbb{T}^{\nu}$ оператор $h(k)$ имеет собственное значение ниже порога существенного спектра.

Для дискретного оператора Шредингера $h_{\mu}(k)$, ассоциированного с гамильтонианом системы двух одинаковых частиц (бозонов), взаимодействующих с помощью контактного потенциала притяжения (см. [5]), показано, что он либо имеет единственное собственное значение, либо не имеет собственных значений в зависимости от значений энергии взаимодействия $\mu>0$ и квазиимпульса $k \in \mathbb{T}^{3}$. В случае двух бозонов, взаимодействующих на ближайших соседних узлах [6], найдено точное число собственных значений соответствующего двухчастичного оператора Шредингера на решетке $\mathbb{Z}^{\nu}, \nu=1,2$.

В настоящей работе рассматривается гамильтониан $\hat{h}_{\mu}$ системы двух фермионов, движущихся на $\nu$-мерной решетке $\mathbb{Z}^{\nu}, \nu \geqslant 1$, и взаимодействующих на ближайших соседних узлах. Для $\nu$-мерного $(\nu \geqslant 1)$ дискретного двухчастичного оператора Шредингера $h_{\mu}(k)$, ассоциированного с гамильтонианом $\hat{h}_{\mu}$, установлены число и местоположение собственных значений в зависимости от параметров оператора значений энергии взаимодействия на соседних узлах $\mu>0$ и полного квазиимпульса системы $k \in \mathbb{T}^{\nu}$. В частности, показано, что для некоторого $\mu=\mu_{0}(\nu)>0$ нижний порог существенного спектра является $\nu$-кратным виртуальным уровнем (при $\nu=1,2$ ) или $\nu$-кратным собственным значением (при $\nu \geqslant 3$ ) оператора $h_{\mu_{0}}(\mathbf{0})$. Следовательно, доказано, что при всех ненулевых значениях $k \in \mathbb{T}^{\nu}$ оператор $h_{\mu_{0}}(k)$ имеет ровно $\nu$ собственных значений ниже порога существенного спектра.

\section{2. ГАМИЛЬТОНИАН СИСТЕМЫ ДВУХ ФЕРМИОНОВ В КООРДИНАТНОМ И ИМПУЛЬСНОМ ПРЕДСТАВЛЕНИЯХ}

Пусть $\mathbb{Z}^{\nu}$ - $\nu$-мерная целочисленная решетка, $\ell_{2}\left(\left(\mathbb{Z}^{\nu}\right)^{2}\right)$ - гильбертово пространство квадратично-суммируемых функций, $\ell_{2}^{\text {as }}\left(\left(\mathbb{Z}^{\nu}\right)^{2}\right) \subset \ell_{2}\left(\left(\mathbb{Z}^{\nu}\right)^{2}\right)$ - подпространство антисимметричных функций. В координатном представлении гамильтониан системы двух фермионов с массой $m=1$ действует в гильбертовом пространстве $\ell_{2}^{\text {as }}\left(\left(\mathbb{Z}^{\nu}\right)^{2}\right)$ по формуле

$$
\begin{gathered}
\hat{h}_{\mu}=\hat{h}^{0}-\mu \hat{v}, \\
\left(\hat{h}^{0} \hat{\psi}\right)\left(x_{1}, x_{2}\right)= \\
\frac{1}{2} \sum_{|s|=1}\left[2 \hat{\psi}\left(x_{1}, x_{2}\right)-\hat{\psi}\left(x_{1}+s, x_{2}\right)-\hat{\psi}\left(x_{1}, x_{2}+s\right)\right], \quad \hat{\psi} \in \ell_{2}^{\text {as }}\left(\left(\mathbb{Z}^{\nu}\right)^{2}\right), \\
(\hat{v} \hat{\psi})\left(x_{1}, x_{2}\right)=\hat{v}\left(x_{1}-x_{2}\right) \hat{\psi}\left(x_{1}, x_{2}\right), \quad \hat{\psi} \in \ell_{2}^{\text {as }}\left(\left(\mathbb{Z}^{\nu}\right)^{2}\right),
\end{gathered}
$$


где

$$
\hat{v}(x)=\left\{\begin{array}{lll}
1 / 2 & \text { при } & |x|=1 \\
0 & \text { при } & |x| \neq 1
\end{array}\right.
$$

$|x|=\left|x^{(1)}\right|+\left|x^{(2)}\right|+\cdots+\left|x^{(\nu)}\right|, x=\left(x^{(1)}, x^{(2)}, \ldots, x^{(\nu)}\right) \in \mathbb{Z}^{\nu}, \mu>0$ - энергия взаимодействия двух частиц на ближайших соседних узлах. Отметим, что двухчастичный гамильтониан $\hat{h}_{\mu}$ является ограниченным самосопряженным оператором в $\ell_{2}^{\text {as }}\left(\left(\mathbb{Z}^{\nu}\right)^{2}\right)$.

Пусть $\mathbb{T}^{\nu}-\nu$-мерный тор, т.е. куб $(-\pi, \pi]^{\nu}$ с соответствующим отождествлением противоположных граней. Он рассматривается как абелева группа, в которой операции сложения и умножения на вещественное число введены так же, как в $\mathbb{R}^{\nu}$, но по модулю $(2 \pi \mathbb{Z})^{\nu}$. Пусть $L_{2}\left(\mathbb{T}^{\nu}\right)$ - гильбертово пространство квадратично-интегрируемых функций, определенных на $\mathbb{T}^{\nu}, L_{2}^{\circ}\left(\mathbb{T}^{\nu}\right) \subset L_{2}\left(\mathbb{T}^{\nu}\right)$ - подпространство нечетных функций.

После преобразования Фурье и выделения полного квазиимпульса $k \in \mathbb{T}^{\nu}$ системы (см. [4], [5]) изучение спектральных свойств оператора $\hat{h}_{\mu}$ сводится к изучению семейства двухчастичных дискретных операторов Шредингера $h_{\mu}(k), k \in \mathbb{T}^{\nu}$, действующих в $L_{2}^{\circ}\left(\mathbb{T}^{\nu}\right)$ по формуле

$$
h_{\mu}(k)=h^{0}(k)-\mu v .
$$

Здесь невозмущенный оператор $h^{0}(k)$ есть оператор умножения на функцию:

$$
\left(h^{0}(k) f\right)(q)=E_{k}(q) f(q), \quad f \in L_{2}^{\mathrm{o}}\left(\mathbb{T}^{\nu}\right),
$$

где

$$
E_{k}(q)=2 \sum_{j=1}^{\nu}\left(1-\cos \frac{k^{(j)}}{2} \cos q^{(j)}\right)
$$

Оператор взаимодействия (возмущения) $v$ является оператором ранга $\nu \geqslant 1$ и действует в гильбертовом пространстве $L_{2}^{\mathrm{o}}\left(\mathbb{T}^{\nu}\right)$ по формуле

$$
(v f)(q)=\frac{1}{(2 \pi)^{\nu}} \sum_{i=1}^{\nu} \sin q^{(i)} \int_{\mathbb{T}^{\nu}} \sin t^{(i)} f(t) d t .
$$

\section{3. СУЩЕСТВЕННЫЙ СПЕКТР И ВИРТУАЛЬНЫЙ УРОВЕНЬ}

Возмущение $v$ оператора $h^{0}(k)$ является оператором ранга $\nu$. Следовательно, согласно теореме Вейля о сохранении существенного спектра при компактном возмущении (см. [7]) существенный спектр $\sigma_{\text {ess }}\left(h_{\mu}(k)\right)$ оператора $h_{\mu}(k)$ совпадает со спектром оператора $h^{0}(k)$. Поскольку $h^{0}(k)$ есть оператор умножения на функцию $E_{k}(q)$, имеем

$$
\sigma_{\mathrm{ess}}\left(h_{\mu}(k)\right)=\left[E_{\min }(k), E_{\max }(k)\right]
$$


где

$$
\begin{aligned}
& E_{\min }(k)=\min _{q \in \mathbb{T}^{\nu}} E_{k}(q)=2 \sum_{j=1}^{\nu}\left(1-\cos \frac{k^{(j)}}{2}\right) \geqslant 0, \\
& E_{\max }(k)=\max _{q \in \mathbb{T}^{\nu}} E_{k}(q)=2 \sum_{j=1}^{\nu}\left(1+\cos \frac{k^{(j)}}{2}\right) \leqslant 4 \nu .
\end{aligned}
$$

ЗАмечАниЕ 1 . При $k=\tilde{\pi}=(\pi, \pi, \ldots, \pi) \in \mathbb{T}^{\nu}$ существенный спектр $\sigma_{\mathrm{ess}}\left(h_{\mu}(k)\right)$ оператора $h_{\mu}(k)$ вырождается, т.е. превращается в точку $E_{\min }(\tilde{\pi})=E_{\max }(\tilde{\pi})=\{2 \nu\}$, поэтому $\sigma_{\text {ess }}\left(h_{\mu}(k)\right)$ не является абсолютно непрерывным при любом $k \in \mathbb{T}^{\nu}$.

Для каждого $k \in(-\pi, \pi)^{\nu}$ и $z \leqslant E_{\min }(k)$ в $L_{2}^{\mathrm{o}}\left(\mathbb{T}^{\nu}\right)$ определим интегральный оператор Бирмана-Швингера $G_{\mu}(k, z)$ с ядром

$$
G_{\mu}(k, z ; p, q)=\frac{2 \mu}{(2 \pi)^{2 \nu}} \sum_{i=1}^{\nu} \sin p^{(i)} \sin q^{(i)} \int_{\mathbb{T}^{\nu}} \frac{\sin ^{2} t^{(i)} d t}{E_{k}(t)-z} .
$$

Отметим, что для любого $z<E_{\min }(k)$ имеет место равенство

$$
G_{\mu}(k, z)=\mu v^{1 / 2} r_{0}(k, z) v^{1 / 2},
$$

где $v^{1 / 2}$ - положительный корень положительного оператора $v, r_{0}(k, z)$ - резольвента оператора $h^{0}(k)$.

ОПРЕДЕЛЕНИЕ. Пусть $\nu=1$ или $\nu=2$. Будем говорить, что оператор $h_{\mu}(\mathbf{0})$ имеет $\nu$-кратный виртуальный уровень на пороге $E_{\min }(\mathbf{0})=0, \mathbf{0}=(0,0, \ldots, 0) \in \mathbb{T}^{\nu}$, если число 1 является $\nu$-кратным собственным значением оператора $G_{\mu}(\mathbf{0}, 0)$ (см. ниже лемму 5).

ЗАМЕЧАНИЕ 2. Пусть $\nu=1$ или $\nu=2$, число 1 является $\nu$-кратным собственным значением оператора $G_{\mu}(\mathbf{0}, 0)$ и $L^{(\nu)} \subset L_{2}^{\mathrm{o}}\left(\mathbb{T}^{\nu}\right)$ - подпространство, натянутое на соответствующие собственные функции $\psi_{i}, i=1,2, \ldots, \nu$. Тогда для каждого $\psi \in L^{(\nu)}$ функция

$$
f(q)=\frac{\mu\left(v^{1 / 2} \psi\right)(q)}{E_{\mathbf{0}}(q)}=\frac{\mu}{E_{\mathbf{0}}(q)} \frac{\sqrt{2}}{(2 \pi)^{\nu}} \sum_{i=1}^{\nu} \sin q^{(i)} \int_{\mathbb{T}^{\nu}} \sin t^{(i)} \psi(t) d t
$$

удовлетворяет уравнению Шредингера $h_{\mu}(\mathbf{0}) f=0$ и при этом $f \notin L_{2}^{\mathrm{o}}\left(\mathbb{T}^{\nu}\right)$.

\section{4. ФОРМУЛИРОВКА ОСНОВНЫХ РЕЗУЛЬТАТОВ}

Заметим, что функция $E_{\mathbf{0}}(q)=E_{\mathbf{0}}\left(q^{(1)}, q^{(2)}, \ldots, q^{(\nu)}\right)$ имеет невырожденный минимум в нуле и инвариантна относительно перестановки аргументов $q^{(1)}, q^{(2)}, \ldots, q^{(\nu)}$. Следовательно, интеграл

$$
\int_{\mathbb{T}^{\nu}} \frac{\sin ^{2} q^{(i)}}{E_{\mathbf{0}}(q)} d q
$$

существует и не зависит от выбора $i=\overline{1, \nu}$. 
Пусть

$$
\mu_{0}=(2 \pi)^{\nu}\left(\int_{\mathbb{T}^{\nu}} \frac{\sin ^{2} q^{(i)}}{E_{\mathbf{0}}(q)} d q\right)^{-1}, \quad i=\overline{1, \nu} .
$$

Основным результатом работы является следующая теорема, которая устанавливает местоположение, число собственных значений и виртуальных уровней оператора $h_{\mu}(k)$.

Теорема. 1. Пусть $0<\mu<\mu_{0}$. Тогда существует такое разбиение тора $\mathbb{T}^{\nu}$ на непустые непересекаюшиеся множества $\left\{G_{\mu}^{(l)}\right\}, l=\overline{0, \nu}$, что при $k \in G_{\mu}^{(l)}, l=\overline{1, \nu}$, оператор $h_{\mu}(k)$ имеет только $l$ положительных собственных значений $z_{\mu}^{(i)}(k)$, $i=\overline{1, l},\left(\right.$ с учетом кратности) ниже порога $E_{\min }(k)$, a nри $k \in G_{\mu}^{(0)}$ оператор $h_{\mu}(k)$ не имеет собственных значений ниже порога $E_{\min }(k)$ существенного спектра.

2. Пусть $\mu=\mu_{0}$. При $\nu=1$ или $\nu=2$ оператор $h_{\mu}(\mathbf{0})$ имеет $\nu$-кратный виртуальный уровенъ на пороге $E_{\min }(\mathbf{0})=0$. При $\nu \geqslant 3$ порог $E_{\min }(\mathbf{0})=0$ является $\nu$-кратным собственным значением оператора $h_{\mu}(\mathbf{0})$. Для любых $\nu \in \mathbb{N} u k \in \mathbb{T}^{\nu}$, $k \neq \mathbf{0}$, оператор $h_{\mu}(k)$ имеет ровно $\nu$ собственных значений $z_{\mu}^{(i)}(k), i=\overline{1, \nu}$, (с учетом кратности) ниже порога $E_{\min }(k) u z_{\mu}^{(i)}(k)>0, i=\overline{1, \nu}$.

3. Пусть $\mu>\mu_{0}$. Тогда для любого $k \in \mathbb{T}^{\nu}$ оператор $h_{\mu}(k)$ имеет ровно $\nu$ собственных значений $z_{\mu}^{(i)}(k), i=\overline{1, \nu}$, ниже порога $E_{\min }(k)$. При $\mu>4 \nu$ имеют место неравенства $z_{\mu}^{(i)}(k)<0, i=\overline{1, \nu}$.

ЗАмЕчаниЕ 3. Так как возмущенный оператор $v$ положителен, $h_{\mu}(k)$ не имеет собственных значений, лежащих правее $E_{\max }(k)$.

\section{5. СВЕДЕНИЕ К ИССЛЕДОВАНИЮ НУЛЕЙ ОПРЕДЕЛИТЕЛЯ ФРЕДГОЛЬМА}

Пусть $\mathbb{C}$ - комплексная плоскость. Для каждого $k \in \mathbb{T}^{\nu}$ и $\mu>0$ определим в $\mathbb{C} \backslash\left[E_{\min }(k), E_{\min }(k)\right]$ функцию

$$
\Delta_{\mu}^{(i)}(k, z)=1-\frac{\mu}{(2 \pi)^{\nu}} \int_{\mathbb{T}^{\nu}} \frac{\sin ^{2} q^{(i)}}{E_{k}(q)-z} d q, \quad i=\overline{1, \nu} .
$$

Обозначим через $\Delta_{\mu}(k, z)$ определитель Фредгольма оператора $I-G_{\mu}(k, z)$.

Лемма 1. Верно следующее равенство:

$$
\Delta_{\mu}(k, z)=\prod_{i=1}^{\nu} \Delta_{\mu}^{(i)}(k, z) .
$$

ДоказАтельство. Оператор $G_{\mu}(k, z)$ отображает все пространство $L_{2}^{\mathrm{o}}\left(\mathbb{T}^{\nu}\right)$ в подпространство $L^{(\nu)}$, натянутое на функции $\sin p^{(i)}, i=1,2, \ldots, \nu$. Пространство $L^{(\nu)}$ является инвариантным относительно оператора $G_{\mu}(k, z)$. Если учесть, что

$$
\begin{gathered}
\int_{\mathbb{T}^{\nu}} \sin t^{(i)} \sin t^{(j)} d t=0, \quad i \neq j, \quad i, j=\overline{1, \nu}, \\
\int_{\mathbb{T}^{\nu}} \sin ^{2} s^{(i)} d s=\frac{(2 \pi)^{\nu}}{2}, \quad i=\overline{1, \nu},
\end{gathered}
$$


то из определения оператора $G_{\mu}(k, z)$ следует, что сужение $\left.G_{\mu}(k, z)\right|_{L^{(\nu)}}$ оператора $G_{\mu}(k, z)$ на $L^{(\nu)}$ представляется в виде матрицы

$$
\left.G_{\mu}(k, z)\right|_{L^{(\nu)}}=\left(a_{\mu}^{i j}(k, z)\right)_{i, j=1}^{\nu},
$$

где $a_{\mu}^{i j}(k, z)=0$ при $i \neq j$ и

$$
a_{\mu}^{i i}(k, z)=\frac{\mu}{(2 \pi)^{\nu}} \int_{\mathbb{T}^{\nu}} \frac{\sin ^{2} t^{(i)} d t}{E_{k}(t)-z} .
$$

Отсюда вытекает доказываемое равенство для определителя оператора $\left.I\right|_{L^{(\nu)}}$ $\left.G_{\mu \lambda}(z)\right|_{L^{(\nu)}}$, где $\left.I\right|_{L^{(\nu)}}-$ единичный оператор в $L^{(\nu)}$.

Следующая лемма вытекает из принципа Бирмана-Швингера и теоремы Фредгольма.

Лемма 2. Для любого $k \in \mathbb{T}^{\nu} u \mu>0$ точка $z \in \mathbb{C} \backslash\left[E_{\min }(k), E_{\min }(k)\right]$ является собственным значением оператора $h_{\mu}(k)$ тогда и только тогда, когда

$$
\Delta_{\mu}(k, z)=0 .
$$

ЛЕмма 3. Для любого $k \in \mathbb{T}^{\nu}$ бункиия $\Delta_{\mu}^{(i)}(k, \cdot)$ является монотонно убываящей на интервале $\left(-\infty, E_{\min }(k)\right) u \Delta_{\mu}^{(i)}(k, z) \rightarrow 1$ nри $z \rightarrow-\infty, i=\overline{1, \nu}$.

ДокАЗАтЕЛьство. При каждом $k \in \mathbb{T}^{\nu}$ функция $\Delta_{\mu}^{(i)}(k, z), i=\overline{1, \nu}$, является аналитической функцией от $z$ в $\mathbb{C} \backslash\left[E_{\min }(k), E_{\min }(k)\right]$ и ее производная по $z$ является отрицательной, т.е.

$$
\frac{\partial \Delta_{\mu}^{(i)}(k, z)}{\partial z}=-\frac{\mu}{(2 \pi)^{\nu}} \int_{\mathbb{T}^{\nu}} \frac{\sin ^{2} q^{(i)}}{\left(E_{k}(q)-z\right)^{2}} d q<0, \quad z \in\left(-\infty, E_{\min }(k)\right) .
$$

Следовательно, для каждого $k \in \mathbb{T}^{\nu}$ функция $\Delta_{\mu}^{(i)}(k, \cdot), i=\overline{1, \nu}$, является монотонно убывающей на интервале $\left(-\infty, E_{\min }(k)\right)$. Очевидно, что $\Delta_{\mu}^{(i)}(k, z) \rightarrow 1$ при $z \rightarrow-\infty$.

Из леммы 3 следует, что существует конечный или бесконечный предел

$$
\lim _{z \rightarrow E_{\min }(k)-0} \Delta_{\mu}^{(i)}(k, z)=\Delta_{\mu}^{(i)}\left(k, E_{\min }(k)\right)=1-\frac{\mu}{(2 \pi)^{\nu}} \int_{\mathbb{T}^{\nu}} \frac{\sin ^{2} p^{(i)}}{E_{k}(p)-E_{\min }(k)} d p, \quad i=\overline{1, \nu} .
$$

Пусть $\Pi=\left\{k=\left(k^{(1)}, k^{(1)}, \ldots, k^{(\nu)}\right) \in \mathbb{T}^{\nu}: k^{(j)}=\pi\right.$ для некоторого $\left.j=1,2, \ldots, \nu\right\}-$ граница куба $(-\pi, \pi]^{\nu}$.

ЗАмечАниЕ 4. Для любого $k \in \mathbb{T}^{\nu} \backslash \Pi$ функция $E_{k}(p)$ имеет единственный невырожденный минимум в нуле, и по теореме Лебега о предельном переходе под знаком интеграла имеем $\Delta_{\mu}^{(i)}\left(k, E_{\min }(k)\right)<\infty, k \in \mathbb{T}^{\nu} \backslash \Pi, i=\overline{1, \nu}$.

При $k \in \Pi$ может выполняться и равенство

$$
\lim _{z \rightarrow E_{\min }(k)-0} \Delta_{\mu}^{(i)}(k, z)=\Delta_{\mu}^{(i)}\left(k, E_{\min }(k)\right)=-\infty, \quad i=\overline{1, \nu}
$$

в зависимости от размерности $\nu \geqslant 1$. 
Лемма 4. 1. Функиия $\Delta_{\mu}^{(i)}\left(k, E_{\min }(k)\right), i=\overline{1, \nu}$, четна по каждой переменной $k^{(j)} \in(-\pi, \pi)$ и монотонно убъвает по $k^{(j)} \in[0, \pi), j=\overline{1, \nu}$.

2. Функиия $\Delta_{\mu}^{(i)}(k, z), i=\overline{1, \nu}$, четна по каждой переменной $k^{(j)} \in \mathbb{T}^{1}$ и при каждом фиксированном $z \leqslant 0$ монотонно возрастает по $k^{(j)} \in[0, \pi], j=\overline{1, \nu}$. При этом имеют место равенства

$$
\begin{aligned}
\max _{k \in \mathbb{T}^{\nu}} \Delta_{\mu}^{(i)}\left(k, E_{\min }(k)\right) & =\min _{k \in \mathbb{T}^{\nu}} \Delta_{\mu}^{(i)}(k, 0)=\Delta_{\mu}^{(i)}(\mathbf{0}, 0), \\
\max _{k \in \mathbb{T}^{\nu}} \Delta_{\mu}^{(i)}(k, 0) & =1-\frac{\mu}{4 \nu}, \quad i=\overline{1, \nu} .
\end{aligned}
$$

ДокАЗАтЕльство. 1. Пользуясь выражением $(1)$, представим функцию $\Delta_{\mu}^{(i)}(k$, $\left.E_{\min }(k)\right)$ в виде

$$
\Delta_{\mu}^{(i)}\left(k, E_{\min }(k)\right)=1-\frac{\mu}{(2 \pi)^{\nu}} \int_{\mathbb{T}^{\nu}} \frac{\sin ^{2} p^{(i)} d p}{2 \sum_{j=1}^{\nu} \cos \left(k^{(j)} / 2\right)\left(1-\cos p^{(j)}\right)} .
$$

Из (3) непосредственно вытекает доказательство утверждения 1 леммы.

2. Функция $E(k ; q):=E_{k}(q)$ является четной по каждой переменной $k^{(j)} \in \mathbb{T}^{1}$, $j=\overline{1, \nu}$, и, следовательно, функция $\Delta_{\mu}^{(i)}(k, z), i=\overline{1, \nu}$, является четной по каждой переменной $k^{(j)} \in \mathbb{T}^{1}, j=\overline{1, \nu}$.

В силу (2) получаем

$$
\frac{\partial \Delta_{\mu}^{(i)}(k, z)}{\partial k^{(j)}}=\frac{\mu \sin \left(k^{(j)} / 2\right)}{(2 \pi)^{\nu}} \int_{\mathbb{T}^{\nu}} \frac{\sin ^{2} q^{(i)} \cos q^{(j)} d q}{\left(E_{k}(q)-z\right)^{2}} .
$$

Пусть $i \neq j$. Выражение $B=E_{k}(q)+2 \cos \left(k^{(j)} / 2\right) \cos q^{(j)}-z>0$ не зависит от компоненты $q^{(j)}$ вектора $q \in \mathbb{T}^{\nu}$. Представим интеграл в правой части равенства (4) в виде

$$
\begin{aligned}
\int_{\mathbb{T}^{\nu}} \frac{\sin ^{2} q^{(i)} \cos q^{(j)} d q}{\left(E_{k}(q)-z\right)^{2}}= & \int_{\mathbb{T}^{\nu-1}}\left(\int_{-\pi}^{\pi} \frac{\cos q^{(j)} d q^{(j)}}{\left(B-2 \cos \left(k^{(j)} / 2\right) \cos q^{(j)}\right)^{2}}\right) \times \\
& \times \sin ^{2} q^{(i)} d q^{(1)} \ldots d q^{(j-1)} d q^{(j+1)} \ldots d q^{(\nu)} .
\end{aligned}
$$

Внутренний интеграл в правой части равенства (5) представим как сумму двух интегралов по отрезкам $[-\pi, 0]$ и $[0, \pi]$. Делая замену переменного $q^{(j)}=t^{(j)}-\pi$ в первом интеграле, получаем

$$
\begin{aligned}
\int_{-\pi}^{\pi} & \frac{\cos q^{(j)} d q^{(j)}}{\left(B-2 \cos \left(k^{(j)} / 2\right) \cos q^{(j)}\right)^{2}}= \\
& =\int_{0}^{\pi} \frac{8 B \cos \left(k^{(j)} / 2\right) \cos ^{2} t^{(j)} d t^{(j)}}{\left(B+2 \cos \left(k^{(j)} / 2\right) \cos t^{(j)}\right)^{2}\left(B-2 \cos \left(k^{(j)} / 2\right) \cos t^{(j)}\right)^{2}} .
\end{aligned}
$$

Из неотрицательности подынтегральной функции при $k^{(j)} \in[0, \pi)$ вытекает, что выражение в правой части равенства (6) положительное. Учитывая соотношения (4)-(6), получаем

$$
\frac{\partial \Delta_{\mu}^{(i)}(k, z)}{\partial k^{(j)}}>0, \quad i, j=\overline{1, \nu}, \quad i \neq j .
$$


Аналогичным рассуждением можно убедиться, что это неравенство верно и при $i=j$. Таким образом, функция $\Delta_{\mu}^{(i)}(k, z), i=\overline{1, \nu}$, строго возрастает по каждому аргументу $k^{(j)} \in[0, \pi]$, поэтому

$$
\min _{k \in \mathbb{T}^{\nu}} \Delta_{\mu}^{(i)}(k, 0)=\Delta_{\mu}^{(i)}(\mathbf{0}, 0), \quad i=\overline{1, \nu}
$$

После элементарных вычислений получаем

$$
\max _{k \in \mathbb{T}^{\nu}} \Delta_{\mu}^{(i)}(k, 0)=1-\frac{\mu}{4 \nu}, \quad i=\overline{1, \nu} .
$$

ЛЕмма 5. Следующие утверждения эквивалентны:

а) при $\nu=1$ или $\nu=2$ число $z=0$ является $\nu$-кратным виртуальным уровнем (и соответственно $\nu$-кратным собственным значением при $\nu \geqslant 3$ ) оператоpa $h_{\mu}(\mathbf{0})$;

б) $\Delta_{\mu}^{(i)}(\mathbf{0}, 0)=0$ для всех $i=\overline{1, \nu}$;

в) $\Delta_{\mu}^{(i)}(\mathbf{0}, 0)=0$ для некоторого $i=\overline{1, \nu}$;

г) $\mu=\mu_{0}$.

Доказательство. Достаточно доказать, что а) $\Rightarrow$ б) $\Rightarrow$ в) $\Rightarrow$ г) $\Rightarrow$ a).

Пусть $\nu=1$ или $\nu=2$. Согласно определению число 1 является $\nu$-кратным собственным значением оператора $G_{\mu}(\mathbf{0}, 0)$, т.е. уравнения

$$
G_{\mu}(\mathbf{0}, 0) \psi_{i}=\psi_{i}, \quad i=\overline{1, \nu}
$$

имеют ненулевые ортогональные решения $\psi_{i} \in L_{2}^{\mathrm{o}}\left(\mathbb{T}^{\nu}\right), i=\overline{1, \nu}$.

Учитывая равенства $\int_{\mathbb{T}^{\nu}} \sin t^{(j)} d t=0, j=\overline{1, \nu}$, получаем систему уравнений, эквивалентную (7):

$$
\psi_{i}(q)=\frac{2 \mu c_{i}}{(2 \pi)^{2 \nu}} \sum_{j=1}^{\nu} \sin q^{(j)} \int_{\mathbb{T}^{\nu}} \frac{\sin ^{2} t^{(j)} d t}{E_{\mathbf{0}}(t)}
$$

где

$$
c_{i}=\int_{\mathbb{T}^{\nu}} \sin t^{(i)} \psi_{i}(t) d t, \quad i=\overline{1, \nu}
$$

Подставляя правую часть равенства (8) в $(9)$, получаем равенства $c_{i} \Delta_{\mu}^{(i)}(\mathbf{0}, 0)=0$, $i=\overline{1, \nu}$, откуда вытекает утверждение б) леммы.

Пусть $\nu \geqslant 3$ и число $z=0$ является $\nu$-кратным собственным значением оператора $h_{\mu}(\mathbf{0})$. Тогда $f \in L_{2}^{\mathrm{o}}\left(\mathbb{T}^{\nu}\right)$ из подпространства собственных функций, соответствующих $\nu$-кратному собственному значению $z=0$, имеет вид

$$
f(q)=\sum_{i=1}^{\nu} c_{i} \varphi_{i}(q)
$$

где

$$
c_{i}=\frac{\mu}{(2 \pi)^{\nu}} \int_{\mathbb{T}^{\nu}} \sin t^{(i)} f(t) d t, \quad \varphi_{i}(q)=\frac{\sin q^{(i)}}{E_{\mathbf{0}}(q)}, \quad i=\overline{1, \nu}
$$


Поскольку функция $E_{\mathbf{0}}(q)=E_{\mathbf{0}}\left(q^{(1)}, q^{(2)}, \ldots, q^{(\nu)}\right)$ четна по каждой переменной $q^{(i)} \in \mathbb{T}^{1}$, имеет невырожденный минимум в нуле и $\sin q^{(i)} \in L_{2}^{\mathrm{o}}\left(\mathbb{T}^{\nu}\right)$, то $\varphi_{i}(q) \in$ $L_{2}^{\mathrm{o}}\left(\mathbb{T}^{\nu}\right), i=\overline{1, \nu}$, при $\nu \geqslant 3$ и

$$
\left(\varphi_{i}, \varphi_{j}\right)=0, \quad \int_{\mathbb{T}^{\nu}} \sin t^{(j)} \varphi_{i}(t) d t=0, \quad i \neq j, \quad i, j=\overline{1, \nu}
$$

Подставляя (10) в первое равенство (11) и учитывая (12), получаем

$$
c_{i} \Delta_{\mu}^{(i)}(\mathbf{0}, 0)=0, \quad i=\overline{1, \nu} .
$$

По условию $h_{\mu}(\mathbf{0})$ имеет $\nu$-кратное собственное значение $z=0$, и, следовательно, система уравнений (13) имеет $\nu$ нетривиальных решений тогда и только тогда, когда $\Delta_{\mu}^{(i)}(\mathbf{0}, 0)=0$ при всех $i=\overline{1, \nu}$.

Если равенство $\Delta_{\mu}^{(i)}(\mathbf{0}, 0)=0$ выполняется при всех $i=\overline{1, \nu}$, то $\Delta_{\mu}^{(i)}(\mathbf{0}, 0)=0$ и для некоторого $i=\overline{1, \nu}$. Поскольку функция $E_{\mathbf{0}}(q)=E_{\mathbf{0}}\left(q^{(1)}, q^{(2)}, \ldots, q^{(\nu)}\right)$ инвариантна относительно перестановки аргументов $q^{(1)}, q^{(2)}, \ldots, q^{(\nu)}$, верно и обратное утверждение.

Поэтому если $\Delta_{\mu}^{(i)}(\mathbf{0}, 0)=0$ для некоторого $i=\overline{1, \nu}$, то из (2) и определения числа $\mu_{0}$ получаем, что $\mu=\mu_{0}$.

Пусть $\mu=\mu_{0}$ и $\nu=1$ или $\nu=2$. Тогда ортогональные функции $\psi_{i}(q)=c_{i} \sin q^{(i)} \in$ $L_{2}^{\mathrm{o}}\left(\mathbb{T}^{\nu}\right), i=\overline{1, \nu}$, удовлетворяют системе уравнений $G_{\mu_{0}}(\mathbf{0}, 0) \psi_{i}=\psi_{i}, i=\overline{1, \nu}$, т.е. число $z=0$ является $\nu$-кратным виртуальным уровнем оператора $h_{\mu_{0}}(\mathbf{0})$.

Если $\nu \geqslant 3$, то элементы ортогональной системы $\left\{\varphi_{i}\right\}_{i=1}^{\nu}$, определенные в (11), принадлежат $L_{2}^{\mathrm{o}}\left(\mathbb{T}^{\nu}\right), i=\overline{1, \nu}$, и удовлетворяют системе уравнений $h_{\mu_{0}}(\mathbf{0}) \varphi_{i}=0$, $i=\overline{1, \nu}$. Действительно, в силу (13) получаем

$h_{\mu_{0}}(\mathbf{0}) \varphi_{i}(p)=\sin p^{(i)}-\frac{\mu}{(2 \pi)^{\nu}} \sin p^{(i)} \int_{\mathbb{T}^{\nu}} \frac{\sin ^{2} t^{(i)} d t}{E_{\mathbf{0}}(t)}=\sin p^{(i)} \Delta_{\mu_{0}}^{(i)}(\mathbf{0}, 0)=0, \quad i=\overline{1, \nu}$.

Лемма доказана.

Для любого $k \in \mathbb{T}^{\nu} \backslash \Pi$ положим

$$
D^{(i)}\left(k^{(1)}, k^{(2)}, \ldots, k^{(\nu)}\right)=\int_{\mathbb{T}^{\nu}} \frac{\sin ^{2} p^{(i)} d p}{2 \sum_{j=1}^{\nu} \cos \left(k^{(j)} / 2\right)\left(1-\cos p^{(j)}\right)}, \quad i=\overline{1, \nu} .
$$

ЛЕмма 6. Функиия $D^{(i)}\left(k^{(1)}, k^{(2)}, \ldots, k^{(\nu)}\right)$ симметрична относительно перестановки любых пар переменных $k^{\left(j_{1}\right)}, k^{\left(j_{2}\right)}, j_{1}, j_{2} \in\{1,2, \ldots, \nu\} \backslash\{i\}$. Кроме того, выполняется равенство

$$
D^{(i)}\left(k^{(1)}, \ldots, k^{(i)}, \ldots, k^{(j)}, \ldots, k^{(\nu)}\right)=D^{(j)}\left(k^{(1)}, \ldots, k^{(j)}, \ldots, k^{(i)}, \ldots, k^{(\nu)}\right)
$$

для любых $i<j, i, j=\overline{1, \nu}$.

ДОказАТЕЛЬСтво. Функция

$$
\sum_{j=1}^{\nu} \cos \frac{k^{(j)}}{2}\left(1-\cos p^{(j)}\right)
$$


симметричная относительно перестановки любых пар переменных $\left(k^{\left(j_{1}\right)}, p^{\left(j_{1}\right)}\right)$ и $\left(k^{\left(j_{2}\right)}, p^{\left(j_{2}\right)}\right), j_{1}, j_{2} \in\{1,2, \ldots, \nu\}$. Отсюда и из определения функции $D^{(i)}\left(k^{(1)}\right.$, $\left.k^{(2)}, \ldots, k^{(\nu)}\right)$ вытекает первое утверждение леммы.

Заменяя переменные интегрирования $p^{(j)}$ на $p^{(i)}$ и $p^{(i)}$ на $p^{(j)}$ в правой части (14), получаем равенство (15).

ЗАмЕчАниЕ 5. Поскольку функция $D^{(i)}\left(k^{(1)}, k^{(2)}, \ldots, k^{(\nu)}\right), i=\overline{1, \nu}$, четна по каждой переменной $k^{(j)} \in(-\pi, \pi), j=\overline{1, \nu}$, достаточно рассмотреть случай $k^{(j)} \in$ $[0, \pi)$.

Лемма 7. Пусть $\nu=2$. Если $k^{(1)}>k^{(2)}, k^{(1)}, k^{(2)} \in[0, \pi)$, то имеет место неравенство $D^{(1)}\left(k^{(1)}, k^{(2)}\right)>D^{(1)}\left(k^{(2)}, k^{(1)}\right)$.

ДокАЗАтЕЛьство. Подынтегральная функция разностной функции $D^{(1)}\left(k^{(1)}\right.$, $\left.k^{(2)}\right)-D^{(1)}\left(k^{(2)}, k^{(1)}\right)$ является четной функцией своих аргументов, поэтому имеет место равенство

$$
D^{(1)}\left(k^{(1)}, k^{(2)}\right)-D^{(1)}\left(k^{(2)}, k^{(1)}\right)=4 \int_{0}^{\pi} \int_{0}^{\pi} \frac{\left(\sin ^{2} p^{(1)}-\sin ^{2} p^{(2)}\right) d p^{(1)} d p^{(2)}}{2 \sum_{j=1}^{2} \cos \left(k^{(j)} / 2\right)\left(1-\cos p^{(j)}\right)} .
$$

Рассмотрим отображения $\varphi_{i}: \mathbb{T}^{2} \rightarrow \mathbb{T}^{2}, i=\overline{1,2}$, определенные по формулам $\varphi_{1}(x, y)=$ $(y, x), \varphi_{2}(x, y)=(\pi-x, \pi-y)$, и пусть $\varphi_{3}=\varphi_{1} \circ \varphi_{2}$.

Введем следующие обозначения:

$$
\begin{aligned}
& W^{(1)}=\{x \in[0, \pi / 2), y \in[0, \pi / 2): x<y\}, \\
& W^{(2)}=\{x \in[\pi / 2, \pi), y \in[0, \pi / 2): x>\pi-y\}, \\
& W_{0}^{(1)}=\{x \in[0, \pi / 2), y \in[0, \pi / 2): x=y\}, \\
& W_{0}^{(2)}=\{x \in[\pi / 2, \pi), y \in[0, \pi / 2): x=\pi-y\} .
\end{aligned}
$$

Тогда верны следующие равенства:

$$
\begin{aligned}
& \left\{(x, y) \in[0, \pi)^{2}: \sin ^{2} x<\sin ^{2} y\right\}=W^{(1)} \cup W^{(2)} \cup \varphi_{2}\left(W^{(1)}\right) \cup \varphi_{2}\left(W^{(2)}\right), \\
& \left\{(x, y) \in[0, \pi)^{2}: \sin ^{2} x=\sin ^{2} y\right\}=W_{0}^{(1)} \cup W_{0}^{(2)} \cup \varphi_{2}\left(W_{0}^{(1)}\right) \cup \varphi_{2}\left(W_{0}^{(2)}\right), \\
& \left\{(x, y) \in[0, \pi)^{2}: \sin ^{2} x>\sin ^{2} y\right\}=\varphi_{1}\left(W^{(1)}\right) \cup \varphi_{1}\left(W^{(2)}\right) \cup \varphi_{3}\left(W^{(1)}\right) \cup \varphi_{3}\left(W^{(2)}\right) .
\end{aligned}
$$

Меры множеств $W_{0}^{(i)}, i=\overline{1,2}$, равны нулю, поэтому после вычислений правая часть равенства (16) принимает вид

$$
\begin{aligned}
& D^{(1)}\left(k^{(1)}, k^{(2)}\right)-D^{(1)}\left(k^{(2)}, k^{(1)}\right)=16 \sum_{j=1}^{2} \cos \frac{k^{(j)}}{2}\left(\cos ^{2} \frac{k^{(2)}}{2}-\cos ^{2} \frac{k^{(1)}}{2}\right) \times \\
& \quad \times \sum_{i=1}^{2} \iint_{W^{(i)}} \frac{\left(\cos ^{2} p^{(2)}-\cos ^{2} p^{(1)}\right)^{2} d p^{(1)} d p^{(2)}}{\left(\sum_{j=1}^{2} \cos \left(k^{(j)} / 2\right)\left(1-\cos p^{(j)}\right)\right)^{2}\left(\sum_{j=1}^{2} \cos \left(k^{(j)} / 2\right)\left(1+\cos p^{(j)}\right)\right)^{2}} .
\end{aligned}
$$

Подынтегральная функция правой части равенства (17) является неотрицательной. Отсюда и из монотонности функции $\cos x$ на $[0, \pi / 2)$ вытекает, что $D^{(1)}\left(k^{(1)}, k^{(2)}\right)-D^{(1)}\left(k^{(2)}, k^{(1)}\right)>0$ при $k^{(1)}>k^{(2)}$. 


\section{6. ДОКАЗАТЕЛЬСТВО ОСНОВНЫХ РЕЗУЛЬТАТОВ}

Для любого $i=\overline{1, \nu}$ положим

$$
F_{\mu}^{(i)}=\left\{k \in \mathbb{T}^{\nu}: \Delta_{\mu}^{(i)}\left(k, E_{\min }(k)\right)<0\right\} .
$$

Лемма 8. При $k \in F_{\mu}^{(i)}$ оператор $h_{\mu}(k)$ имеет собственное значение ниже порога $E_{\min }(k)$.

ДокАЗАТЕльСТво. Пусть $k \in F_{\mu}^{(i)}$. В силу леммы 3 для каждого $k \in \mathbb{T}^{\nu}$ функция $\Delta_{\mu}^{(i)}(k, \cdot)$ является монотонно убывающей на интервале $\left(-\infty, E_{\min }(k)\right)$. Отсюда и из непрерывности $\Delta_{\mu}^{(i)}(k, \cdot)$ на $\left(-\infty, E_{\min }(k)\right)$ следует, что для каждого $k \in F_{\mu}^{(i)}$ cуществует единственное $z_{\mu}^{(i)}(k) \in\left(-\infty, E_{\min }(k)\right)$ такое, что $\Delta_{\mu}^{(i)}\left(k, z_{\mu}^{(i)}(k)\right)=0, i=\overline{1, \nu}$. Из лемм 1 и 2 получаем, что число $z_{\mu}^{(i)}(k)$ является собственным значением оператора $h_{\mu}(k)$.

Множества $G_{\mu}^{(l)}, l=\overline{0, \nu}$, построим следующим образом:

$$
\begin{aligned}
G_{\mu}^{(l)} & =\bigcup_{\left\{j_{1}, j_{2}, \ldots, j_{\nu}\right\}=\{1,2, \ldots, \nu\}} F_{\mu}^{\left(j_{1}\right)} \cap F_{\mu}^{\left(j_{2}\right)} \cap \cdots \\
\cdots \cap F_{\mu}^{\left(j_{l}\right)} \cap\left(\mathbb{T}^{\nu} \backslash\left(F_{\mu}^{\left(j_{l+1}\right)} \cup \cdots \cup F_{\mu}^{\left(j_{\nu}\right)}\right)\right), & \\
G_{\mu}^{(0)} & =\mathbb{T}^{\nu} \backslash\left(\bigcup_{i=1}^{\nu} G_{\mu}^{(i)}\right) .
\end{aligned}
$$

Лемма 9. Пусть $0<\mu<\mu_{0}$. Тогда множества $G_{\mu}^{(l)}, l=\overline{0, \nu}$, являются непустыми.

ДокАЗАТЕльство. Сначала докажем, что множество $G_{\mu}^{(0)}$ содержит точку $k=$ $\mathbf{0} \in \mathbb{T}^{\nu}$. Для этого нужно показать, что $\mathbf{0} \notin G_{\mu}^{(i)}, i=\overline{1, \nu}$. Действительно, учитывая равенство $E_{\min }(\mathbf{0})=0$, согласно определению числа $\mu_{0}$ для любых $0<\mu<\mu_{0}$ и $l=\overline{1, \nu}$ получим, что имеет место неравенство $\Delta_{\mu}^{(l)}(\mathbf{0}, 0)=1-\mu / \mu_{0}>0$. Отсюда вытекает, что $k=\mathbf{0} \in G_{\mu}^{(0)}$.

Докажем, что множества

$$
\begin{array}{rll}
E_{\mu}^{(l)}=\left\{k \in \mathbb{T}^{\nu}: \Delta_{\mu}^{(i)}\left(k, E_{\min }(k)\right)<0\right. & \text { для } \quad i=\overline{1, l} \quad \text { и } \\
\Delta_{\mu}^{(i)}\left(k, E_{\min }(k)\right) \geqslant 0 & \text { для } \quad i=\overline{l+1, \nu}\}
\end{array}
$$

непусты. Тогда множества $G_{\mu}^{(l)}, l=\overline{1, \nu}$, также будут непусты.

Пусть $\mathbb{T}_{(l)}^{\nu}=\left\{k=\left(k^{(1)}, \ldots, k^{(\nu)}\right) \in \mathbb{T}^{\nu}: k^{(1)}=\cdots=k^{(l)} ; k^{(l+1)}=\cdots=k^{(\nu)}\right\}$. Тогда для каждого $k \in \mathbb{T}_{(l)}^{\nu} \backslash \Pi$ функция $\Delta_{\mu}^{(i)}\left(k, E_{\min }(k)\right)$ принимает вид

$$
\Delta_{\mu}^{(i)}\left(k, E_{\min }(k)\right)=1-\frac{\mu}{(2 \pi)^{\nu}} D^{(i)}(\underbrace{k^{(l)}, k^{(l)}, \ldots, k^{(l)}}_{l}, \underbrace{k^{(l+1)}, \ldots, k^{(l+1)}}_{\nu-l}), \quad i=\overline{1, \nu} .
$$


Согласно лемме 6 имеем

$$
\begin{aligned}
& D^{(1)}\left(k^{(l)}, k^{(l)}, \ldots, k^{(l)}, k^{(l+1)}, \ldots, k^{(l+1)}\right)= \\
& \quad=D^{(2)}\left(k^{(l)}, k^{(l)}, \ldots, k^{(l)}, k^{(l+1)}, \ldots, k^{(l+1)}\right)=\ldots \\
& \ldots=D^{(l)}\left(k^{(l)}, k^{(l)}, \ldots, k^{(l)}, k^{(l+1)}, \ldots, k^{(l+1)}\right) \\
& D^{(l+1)}\left(k^{(l)}, k^{(l)}, \ldots, k^{(l)}, k^{(l+1)}, \ldots, k^{(l+1)}\right)= \\
& \quad=D^{(l+2)}\left(k^{(l)}, k^{(l)}, \ldots, k^{(l)}, k^{(l+1)}, \ldots, k^{(l+1)}\right)=\ldots \\
& \ldots=D^{(\nu)}\left(k^{(l)}, k^{(l)}, \ldots, k^{(l)}, k^{(l+1)}, \ldots, k^{(l+1)}\right) .
\end{aligned}
$$

Теперь покажем, что если $k^{(l)}>k^{(l+1)}$, то имеет место неравенство $D^{(l)}\left(k^{(l)}\right.$, $\left.k^{(l)}, \ldots, k^{(l)}, k^{(l+1)}, \ldots, k^{(l+1)}\right)>D^{(l+1)}\left(k^{(l)}, k^{(l)}, \ldots, k^{(l)}, k^{(l+1)}, \ldots, k^{(l+1)}\right)$. Действительно, имеем

$$
\begin{aligned}
& D^{(l)}\left(k^{(l)}, k^{(l)}, \ldots, k^{(l)}, k^{(l+1)}, \ldots, k^{(l+1)}\right)- \\
& \quad-D^{(l+1)}\left(k^{(l)}, k^{(l)}, \ldots, k^{(l)}, k^{(l+1)}, \ldots, k^{(l+1)}\right)= \\
& =\int_{\mathbb{T}^{\nu-2}}\left(\int_{\mathbb{T}^{2}} \frac{\left(\sin ^{2} q^{(l)}-\sin ^{2} q^{(l+1)}\right) d q^{(l)} d q^{(l+1)}}{A(k, q)-2 \cos \left(\frac{k^{(l)}}{2}\right) \cos q^{(l)}-2 \cos \left(\frac{k^{(l+1)}}{2}\right) \cos q^{(l+1)}}\right) d q^{(1)} \ldots d q^{(l-1)} \times \\
& \quad \times d q^{(l+2)} \ldots d q^{(\nu)},
\end{aligned}
$$

где

$$
\begin{aligned}
A(k, q)= & 2 \cos \frac{k^{(l)}}{2} \sum_{\alpha=1}^{l-1}\left(1-\cos q^{(\alpha)}\right)+2 \cos \frac{k^{(l+1)}}{2} \sum_{\alpha=l+2}^{\nu}\left(1-\cos q^{(\alpha)}\right)+ \\
& +2 \cos \frac{k^{(l)}}{2}+2 \cos \frac{k^{(l+1)}}{2}>0 .
\end{aligned}
$$

Действуя так же, как при доказательстве леммы 7, можно показать, что значение внутреннего интеграла в правой части равенства (19) положительно. Отсюда получаем, что $D^{(l)}\left(k^{(l)}, \ldots, k^{(l)}, k^{(l+1)}, \ldots, k^{(l+1)}\right)-D^{(l+1)}\left(k^{(l)}, \ldots, k^{(l)}, k^{(l+1)}, \ldots\right.$ $\left.\ldots, k^{(l+1)}\right)>0$ при $k^{(l)}>k^{(l+1)}$.

Пусть $k \in \mathbb{T}_{(l)}^{\nu} \backslash \Pi$ и $k^{(l)}>k^{(l+1)}$. Из полученных для функций $D^{(l)}\left(k^{(l)}, k^{(l)}, \ldots\right.$ $\left.\ldots, k^{(l)}, k^{(l+1)}, \ldots, k^{(l+1)}\right)$ соотношений получаем следующие равенства:

$$
\begin{aligned}
\Delta_{\mu}^{(1)}\left(k, E_{\min }(k)\right) & =\Delta_{\mu}^{(2)}\left(k, E_{\min }(k)\right)=\cdots=\Delta_{\mu}^{(l)}\left(k, E_{\min }(k)\right), \\
\Delta_{\mu}^{(l+1)}\left(k, E_{\min }(k)\right) & =\Delta_{\mu}^{(l+2)}\left(k, E_{\min }(k)\right)=\cdots=\Delta_{\mu}^{(\nu)}\left(k, E_{\min }(k)\right), \\
\Delta_{\mu}^{(l)}\left(k, E_{\min }(k)\right) & <\Delta_{\mu}^{(l+1)}\left(k, E_{\min }(k)\right) .
\end{aligned}
$$

Кроме того, для любого $0<\mu<\mu_{0}$ и $k \in \mathbb{T}_{(l)}^{\nu}$ имеем

$$
\begin{aligned}
\lim _{k^{(l+1)} \rightarrow \pi} \Delta_{\mu}^{(l+1)}\left(k, E_{\min }(k)\right) & =-\infty \\
\lim _{k^{(l)} \rightarrow 0} \Delta_{\mu}^{(l)}\left(k, E_{\min }(k)\right) & =\Delta_{\mu}^{(l)}(\mathbf{0}, 0)>0 .
\end{aligned}
$$


Отсюда, из компактности множества $\mathbb{T}_{(l)}^{\nu}$ и непрерывности функций $\Delta_{\mu}^{(l)}\left(k, E_{\min }(k)\right)$ для любого $0<\mu<\mu_{0}$ вытекает, что существует $k_{0} \in \mathbb{T}_{(l)}^{\nu}$ такое, что выполняются неравенства $\Delta_{\mu}^{(l)}\left(k_{0}, E_{\min }\left(k_{0}\right)\right)<0$ и $\Delta_{\mu}^{(l+1)}\left(k_{0}, E_{\min }\left(k_{0}\right)\right)>0$ одновременно, т.е. множество $E_{\mu}^{(l)}$ непусто.

ДоказАТЕЛЬСтво теоремы. 1. Множества $k \in G_{\mu}^{(l)}, l=\overline{0, \nu}$, определенные по формулам (18), удовлетворяют требованиям п. 1 теоремы. Действительно, пусть $k \in G_{\mu}^{(l)}, l=\overline{1, \nu}$. Из определения множества $G_{\mu}^{(l)}$ вытекает, что $k \in F_{\mu}^{\left(j_{\alpha}\right)}$ при всех $\alpha=\overline{1, l}$ и $k \notin F_{\mu}^{\left(j_{\alpha}\right)}$ при $\alpha=\overline{l+1, \nu}$ для некоторого $j_{\alpha}$. Согласно определению множества $G_{\mu}^{(l)}$ и леммам 8, 9 заключаем, что при $k \in G_{\mu}^{(l)}, l=\overline{1, \nu}$, оператор $h_{\mu}(k)$ имеет ровно $l$ собственных значений ниже порога $E_{\min }(k)$. Из определения множества $G_{\mu}^{(l)}$, $l=\overline{0, \nu}$, утверждений лемм 1,2 и 9 получаем, что при $k \in G_{\mu}^{(0)}$ оператор $h_{\mu}(k)$ не имеет собственных значений, лежащих ниже порога $E_{\min }(k)$.

В силу леммы 4 для любых $z<0$ и $k \in \mathbb{T}^{\nu}$ имеют место неравенства $\Delta_{\mu}^{(i)}(k, z)>$ $\Delta_{\mu}^{(i)}(\mathbf{0}, z)>\Delta_{\mu}^{(i)}(\mathbf{0}, 0)>0, i=\overline{1, \nu}$. Отсюда и из утверждений лемм 1,2 вытекает, что оператор $h_{\mu}(k)$ не имеет отрицательных собственных значений.

2. Пусть $\nu=1$ или $\nu=2$. Тогда согласно лемме 5 функция

$$
\psi(p)=\sum_{i=1}^{\nu} \sin p^{(i)} \in L_{2}^{\mathrm{o}}\left(\mathbb{T}^{\nu}\right)
$$

удовлетворяет уравнению $G_{\mu_{0}}(\mathbf{0}, 0) \psi=\psi$, т.е. оператор $h_{\mu_{0}}(\mathbf{0})$ имеет $\nu$-кратный виртуальный уровень на пороге $E_{\min }(\mathbf{0})=0$.

Пусть $\nu \geqslant 3$. Из определения числа $\mu_{0}$ имеем $\Delta_{\mu_{0}}^{(i)}(\mathbf{0}, 0)=0, i=\overline{1, \nu}$. В силу леммы 5 порог $E_{\min }(\mathbf{0})=0$ является $\nu$-кратным собственным значением оператоpa $h_{\mu_{0}}(\mathbf{0})$.

Из определений функций $E_{k}(q)$ и $E_{\min }(k)$ вытекает, что при ненулевых $k \in \mathbb{T}^{\nu}$ почти всюду имеет место неравенство $E_{k}(q)-E_{\min }(k)<E_{\mathbf{0}}(q)$. Следовательно, получаем неравенство

$$
\Delta_{\mu_{0}}^{(i)}\left(k, E_{\min }(k)\right)<\Delta_{\mu_{0}}^{(i)}(\mathbf{0}, 0)=0, \quad k \neq 0, \quad i=\overline{1, \nu} .
$$

Согласно лемме 8 при всех ненулевых $k \in \mathbb{T}^{\nu}$ оператор $h_{\mu_{0}}(k)$ имеет ровно $\nu$ собственных значений (с учетом кратности) ниже $E_{\min }(k)$. Теперь покажем положительность собственных значений $z_{\mu_{0}}^{(i)}(k), k \neq 0, i=\overline{1, \nu}$. Так как $\Delta_{\mu_{0}}^{(i)}(\mathbf{0}, 0)=0$, в силу леммы 4 получим, что $\Delta_{\mu_{0}}^{(i)}(k, 0)>0, i=\overline{1, \nu}$, при всех ненулевых $k \in \mathbb{T}^{\nu}$. Из монотонности функции $\Delta_{\mu_{0}}^{(i)}(k, \cdot)$ при всех ненулевых $k \in \mathbb{T}^{\nu}$ вытекают неравенства

$$
\Delta_{\mu_{0}}^{(i)}(k, 0)>\Delta_{\mu_{0}}^{(i)}\left(k, z_{\mu_{0}}^{(i)}(k)\right)=0>\Delta_{\mu}^{(i)}\left(k, E_{\min }(k)\right), \quad i=\overline{1, \nu} .
$$

Таким образом, собственные значения $z_{\mu_{0}}^{(i)}(k), i=\overline{1, \nu}$, оператора $h_{\mu_{0}}(k)$ принадлежат интервалу $\left(0, E_{\min }(k)\right)$.

3. Пусть $\mu>\mu_{0}$. Используя соотношение

$$
\Delta_{\mu}^{(i)}\left(k, E_{\min }(k)\right)<\Delta_{\mu}^{(i)}(\mathbf{0}, 0)<\Delta_{\mu_{0}}^{(i)}(\mathbf{0}, 0)=0
$$


и рассуждая, как в п. 2 доказательства теоремы, убеждаемся, что оператор $h_{\mu}(k)$ имеет ровно $\nu$ собственных значений (с учетом кратности) ниже порога $E_{\min }(k)$. При $\mu>4 \nu$ из утверждений леммы 4 вытекают соотношения

$$
\Delta_{\mu}^{(i)}(k, 0) \leqslant \max _{k \in \mathbb{T}^{\nu}} \Delta_{\mu}^{(i)}(k, 0)=1-\frac{\mu}{4 \nu}<0, \quad i=\overline{1, \nu} .
$$

Согласно лемме 8 оператор $h_{\mu}(k)$ имеет $\nu$ отрицательных собственных значений (с учетом кратности), лежащих ниже порога $E_{\min }(k)$.

Благодарности. Авторы выражают глубокую благодарность Р. А. Минлосу за внимание к работе.

\section{Список литературы}

[1] P. A. Faria da Veiga, L. Ioriatti, M. O'Carroll, Phys. Rev. E, 66:1 (2002), 6130.

[2] D. C. Mattis, Rev. Modern Phys., 58:2 (1986), 361-379.

[3] A. I. Mogilner, "Hamiltonians in solid-state physics as multiparticle discrete Schrödinger operators: problems and results", Many Particle Hamiltonians: Spectra and Scattering, Adv. Soviet Math., 5, ed. R. A. Minlos, AMS, Providence, RI, 1991, 139-194.

[4] S. Albeverio, S. N. Lakaev, K. A. Makarov, Z.I. Muminov, Comm. Math. Phys., 262:1 (2006), 91-115.

[5] С. Н. Лакаев, Функи. анализ и его прил., 27:3 (1993), 15-28.

[6] А. М. Халхужаев, Узбек. матем. журн., 2000, № 3, 32-39.

[7] М. Рид, Б. Саймон, Методы современной математической физики. Т. 4. Анализ операторов, Мир, М., 1982.

Поступила в редакцию 18.03.2008, после доработки 6.05.2008 